\title{
ON GABOR ORTHONORMAL BASES OVER FINITE PRIME FIELDS
}

\author{
A. IOSEVICH, M. KOLOUNTZAKIS, YU. LYUBARSKII, A. MAYELI AND J. PAKIANATHAN
}

\begin{abstract}
We study Gabor orthonormal windows in $L^{2}\left(\mathbb{Z}_{p}^{d}\right)$ for translation and modulation sets $A$ and $B$, respectively, where $p$ is prime and $d \geq 2$. We prove that for a set $E \subset \mathbb{Z}_{p}^{d}$, the indicator function $1_{E}$ is a Gabor window if and only if $E$ tiles and is spectral. Moreover, we prove that for any function $g: \mathbb{Z}_{p}^{d} \rightarrow \mathbb{C}$ with support $E$, if the size of $E$ coincides with the size of the modulation set $B$ or if $g$ is positive, then $g$ is a unimodular function, i.e., $|g|=c 1_{E}$, for some constant $c>0$, and $E$ tiles and is spectral. We also prove the existence of a Gabor window $g$ with full support where neither $|g|$ nor $|\hat{g}|$ is an indicator function and $|B|<<p^{d}$. We conclude the paper with an example and open questions.
\end{abstract}

\section{INTRODUCTION}

In the classical case $\mathbb{R}^{d}$, it is possible to decompose a square integrable function in the Euclidean setting into the infinite sum of translations and modulations of a given function. The function is called window and the family of its translations and modulations is called Gabor system. The Gabor system is a Gabor orthonormal basis if the system is an orthonormal basis. Gabor bases are strong tools in time-frequency analysis of signals (functions). In this paper, we introduce the notion of Gabor orthonormal bases for the finite dimensional vector spaces over finite fields and study their primary properties when the field is a prime filed.

Let $A, B \subset \mathbb{Z}_{p}^{d}$, where $\mathbb{Z}_{p}$ is the cyclic group of size $p$ and $\mathbb{Z}_{p}^{d}$ is the $d$-dimensional vector space over $\mathbb{Z}_{p}$. The purpose of this paper is to investigate Gabor orthonormal bases, also known as Weyl-Heisenberg orthonormal bases for $L^{2}\left(\mathbb{Z}_{p}^{d}\right)$. These are the bases of the form

$$
\mathcal{G}(g, A, B):=\{g(x-a) \chi(x \cdot b)\}_{a \in A ; b \in B},
$$

with window function $g: \mathbb{Z}_{p}^{d} \rightarrow \mathbb{C},\|g\|_{2}=1$ (with respect to the counting measure) and $\chi$ is any non-trivial character of the additive group $\mathbb{Z}_{p}$. The orthogonality is given by

$$
\sum_{x \in \mathbb{Z}_{p}^{d}} g(x-a) \overline{g\left(x-a^{\prime}\right)} \chi\left(x \cdot\left(b-b^{\prime}\right)\right)=0 \text { if }(a, b) \neq\left(a^{\prime}, b^{\prime}\right) .
$$

For convenience we work with $\chi(t)=e^{\frac{2 \pi i t}{p}}$, for $p$ prime, but any non-trivial character will do. The sets $A$ and $B$ are called translation and modulation sets, respectively.

Our investigation is partially motivated by the authors' previous work ([3]) on the Fuglede conjecture in $\mathbb{Z}_{p}^{2}, p$ prime.

Date: August 13, 2018.

The work of the first and fifth listed authors was partially supported by the NSA Grant H98230-15-1-0319. The work of the second author has been supported by grant No 4725 of the University of Crete. The work of fourth author was partially supported by the PSC-CUNY Grant B $\sharp 69625$-00 47 . 
Definition 1.1. We say that $E \subseteq \mathbb{Z}_{p}^{d}$ is spectral if there exists $B \subseteq \mathbb{Z}_{p}^{d}$ such that $L^{2}(E)$ possesses an orthogonal basis of exponentials $\{\chi(x \cdot b)\}_{b \in B}$. In this case we say that $(E, B)$ is a spectral pair. (Note that this is a symmetric relation.)

Definition 1.2. We say that $E \subseteq \mathbb{Z}_{p}^{d}$ tiles by translation if there exists $A \subseteq \mathbb{Z}_{p}^{d}$ such that

$$
\sum_{a \in A} 1_{E}(x-a)=1 \text { for all } x \in \mathbb{Z}_{p}^{d}
$$

In this case we say that $(E, A)$ is a tiling pair. (Again, we note that the tiling pair property is also a symmetric relation.) The set $E$ is packing with $A$ if

$$
\sum_{a \in A} 1_{E}(x-a) \leq 1 \text { for all } x \in \mathbb{Z}_{p}^{d}
$$

In [3] we proved that The Fuglede Conjecture holds for $\mathbb{Z}_{p}^{2}, p$ prime. Indeed:

Theorem 1.3. Let $E \subseteq \mathbb{Z}_{p}^{2}$, p prime. Then $L^{2}(E)$ has an orthogonal basis of characters if and only if $E$ tiles $\mathbb{Z}_{p}^{2}$ by translation.

The Fuglede Conjecture was originally stated in $\mathbb{R}^{d}([2])$. For dimensions $d=3$ and higher the conclusion of Theorem 1.3 is known to be false in $\mathbb{R}^{d}([7,4])$. In $\mathbb{Z}_{p}^{d}$ the conclusion of Theorem 1.3 is known to be false in dimensions four and higher. As of dimension 3 , the implication tile $\rightarrow$ spectral always holds over the finite fields of prime order. It is also well-known that the Fuglede conjecture holds in $\mathbb{Z}_{2}^{3}$ and $\mathbb{Z}_{3}^{3}$ ([1]). It is trivial that the Fuglede conjecture holds in $\mathbb{Z}_{p}, p$ prime.

Our main results follow:

Theorem 1.4. Suppose that $E \subseteq \mathbb{Z}_{p}^{d}$. Then $\left\{|E|^{-1 / 2} 1_{E}(x-a) \chi(x \cdot b)\right\}_{a \in A ; b \in B}$ is an orthonormal basis for $L^{2}\left(\mathbb{Z}_{p}^{d}\right)$ if and only if $(E, B)$ is a spectral pair and $(E, A)$ is a tiling pair.

Our next two results on the characterization of Gabor orthonormal bases in $\mathbb{Z}_{p}^{d}$ are motivated by Liu-Wang's conjecture for the Gabor orthonormal bases in $\mathbb{R}^{d}([5])$.

Theorem 1.5. Let $g \in L^{2}\left(\mathbb{Z}_{p}^{d}\right)$ and let $A \subseteq \mathbb{Z}_{p}^{d}$ and $B \subseteq \mathbb{Z}_{p}^{d}$. Let $E:=\operatorname{supp}(g)$ and $|E|=|B|$. Then $\mathcal{G}(g, A, B)=\{g(x-a) \chi(x \cdot b)\}_{a \in A ; b \in B}$ is orthonormal and complete in $L^{2}\left(\mathbb{Z}_{p}^{d}\right)$ if and only if the following hold.

(i) $|g|=|E|^{-1 / 2} 1_{E}$.

(ii) $(E, B)$ is a spectral pair.

(iii) $(E, A)$ is a tiling pair.

Moreover, if $d=2,1<|A|,|B|<p^{2}$ and $\mathcal{G}(g, A, B)$ is an orthonormal basis for $L^{2}\left(\mathbb{Z}_{p}^{2}\right)$, then $E$ is graph of a function.

We say a set $E \subset \mathbb{Z}_{p}^{2}$ is graph of a function if there is a map $u: \mathbb{Z}_{p} \rightarrow \mathbb{Z}_{p}$ such that $E=$ $\left\{(x, u(x)): u \in \mathbb{Z}_{p}\right\}$.

The following Theorem is similar to Theorem 1.5 but the size assumption on $B$ has been replaced by a positivity assumption.

Theorem 1.6. Let $g \in L^{2}\left(\mathbb{Z}_{p}^{d}\right)$ and let $A \subseteq \mathbb{Z}_{p}^{d}$ and $B \subseteq \mathbb{Z}_{p}^{d}$. Let $E:=\operatorname{supp}(g)$ and $g$ be positive on $E$. Then $\mathcal{G}(g, A, B)$ is an orthonormal basis for $L^{2}\left(\mathbb{Z}_{p}^{d}\right)$ if and only if the following hold true: 
(i) $g=|E|^{-1 / 2} 1_{E}$.

(ii) $(E, B)$ is a spectral pair.

(iii) $(E, A)$ is a tiling pair.

The following result proves that the conclusions $(i)$ - (iii) in Theorems 1.5 and 1.6 generally fail if we remove either the size or the positivity assumption. (See also Example 4.3.)

Here and throughout, given $g: \mathbb{Z}_{p}^{d} \rightarrow \mathbb{C}$, the Fourier transform $\hat{g}$ is defined by

$$
\widehat{g}(m)=p^{-d} \sum_{x \in \mathbb{Z}_{p}^{d}} \chi(-x \cdot m) g(x),
$$

and Plancherel identity is given by

$$
\sum_{m \in \mathbb{Z}_{p}^{d}}|\widehat{g}(m)|^{2}=p^{-d} \sum_{x \in \mathbb{Z}_{p}^{d}}|g(x)|^{2} .
$$

Theorem 1.7. There exists an orthonormal basis $\mathcal{G}(g, A, B)$ with a Gabor window $g$ in $L^{2}\left(\mathbb{Z}_{p}^{d}\right)$, where $g$ has none of the following properties:

- (1) $|g|$ is a constant multiple of an indicator function of a set,

- (2) $|\hat{g}|$ is a constant multiple of an indicator function of a set.

One of the themes of this paper is characterization of window functions, which is one of the subtle problems in the study of Gabor bases. There are only few instances where the characterization can be achieved. The following example is one of those.

Assume that $A$ is a subspace of $\mathbb{Z}_{p}^{d}$. Then for some $1 \leq k<d, A$ is isomorphic with $\mathbb{Z}_{p}^{k} \times\left\{\mathbf{0}_{d-k}\right\}$, where $\mathbf{0}_{d-k}$ is the vector zero in $\mathbb{Z}_{p}^{d-k}$. Let $B$ be the orthogonal complement of $A$. Then $B$ is isomorphic to $\left\{\mathbf{0}_{k}\right\} \times \mathbb{Z}_{p}^{d-k}$. For any $x \in \mathbb{Z}_{p}^{d}$, we denote $x=\left(x_{1}, x_{2}\right) \in \mathbb{Z}_{p}^{k} \times \mathbb{Z}_{p}^{d-k}$ where $x_{1}$ and $x_{2}$ are partitions of $x$ in $\mathbb{Z}_{p}^{d}$. We have the following result.

Theorem 1.8. Let $g \in L^{2}\left(\mathbb{Z}_{p}^{d}\right)$ and $A$ and $B$ be as above. Then the Gabor family $\mathcal{G}(g, A, B)$ is an orthonormal basis for $L^{2}\left(\mathbb{Z}_{p}^{d}\right)$ if and only if the following conditions hold true for any $1 \leq k \leq d$ :

(a) $\left|\sum_{x_{1} \in \mathbb{Z}_{p}^{k}} g\left(x_{1}, x_{2}\right) \chi\left(-x_{1} \cdot m\right)\right|=$ constant $\forall x_{2} \in \mathbb{Z}_{p}^{d-k}$

(b) $\sum_{x_{1} \in \mathbb{Z}_{p}^{k}}|g|^{2}\left(x_{1}, x_{2}\right)=$ constant.

\section{Preliminaries and basic properties of Gabor orthonormal bases in $\mathbb{Z}_{p}^{d}$}

In this section we collect the basic properties of Gabor orthonormal bases in $\mathbb{Z}_{p}^{d}$.

Lemma 2.1. The following hold true.

(i) If the Gabor family (1.1) is an orthonormal basis, then $|A \| B|=p^{d}$.

(ii) If $|A|=1$ and $B=\mathbb{Z}_{p}^{d}$, then for any function $g$ such that $|g(x)|=p^{-d / 2}$, (1.1) is an orthonormal basis.

(iii) If $|B|=1$ and $A=\mathbb{Z}_{p}^{d}$, then for any function $g$ such that $|\widehat{g}(m)|=p^{-d / 2}$, (1.1) is an orthonormal basis.

Proof. The proof is immediate from a direct calculation and basic linear algebra. 
Remark. Notice Lemma 2.1 provides a complete characterization of Gabor orthonormal bases of form (1.1) over the one dimensional space $\mathbb{Z}_{p}$.

Lemma 2.2. Suppose that $g \in L^{2}\left(\mathbb{Z}_{p}^{d}\right)$ and $A, B \subseteq \mathbb{Z}_{p}^{d}$. Then $\mathcal{G}(g, A, B)$ is an orthonormal basis for $L^{2}\left(\mathbb{Z}_{p}^{d}\right)$ if and only if $\mathcal{G}(\widehat{g}, B,-A)$ is an orthonormal basis for $L^{2}\left(\mathbb{Z}_{p}^{d}\right)$.

In the following sections we shall use the above results to construct large families of Gabor orthonormal bases and make connections between this problem and the Fuglede conjecture's result in $\mathbb{Z}_{p}^{2}$ in Theorem 1.3.

\section{Proof of Theorem 1.4}

Proof of Theorem 1.4. Assume that $\mathcal{G}\left(|E|^{-1 / 2} 1_{E}, A, B\right)$ is an orthonormal basis for $L^{2}\left(\mathbb{Z}_{p}^{d}\right)$. Without loss of generality, we assume that $\mathbf{0} \in A$. Then

$$
\sum_{x \in E} \chi\left(x \cdot\left(b-b^{\prime}\right)\right)=0 \text { if } b \neq b^{\prime}, b, b^{\prime} \in B .
$$

Similarly,

$$
\sum_{x \in \mathbb{Z}_{p}^{d}} 1_{E}(x-a) 1_{E}\left(x-a^{\prime}\right)=0 \text { if } a \neq a^{\prime}, a, a^{\prime} \in A
$$

The equation (3.1) implies that $|E| \geq|B|$, while the equation (3.2) implies that $E$ is a packing with $A$, thus $|E| \leq \frac{p^{d}}{|A|}$. Combined with the fact that $|A||B|=p^{d}$ by Lemma 2.1, we see that $|E|=|B|=\frac{p^{d}}{|A|}$. Invoking (3.1) once again, we see that $B$ must be a spectrum for $E$. Invoking (3.2) we see that $A$ must be a tiling set for $E$. The conclusion of Theorem 1.4 for one direction follows.

For the proof of the converse, assume that $(E, A)$ is a tiling pair and $(E, B)$ is a spectral pair. Then $|E||A|=p^{d}$ and $|E|=|B|$. Therefore, $|A||B|=p^{d}$. The orthogonality of the functions in $\left\{|E|^{-1 / 2} 1_{E}(x-a) \chi(x \cdot b)\right\}_{a \in A, b \in B}$ holds by tiling property of $(E, A)$ and spectral property of $(E, B)$. This with the cardinality condition $|A||B|=p^{d}$ completes the proof of the theorem.

\section{Proof of Theorem 1.5}

Let $w: \mathbb{Z}_{p}^{d} \rightarrow[0, \infty)$ and $B \subseteq \mathbb{Z}_{p}^{d}$. We say $B$ is a spectrum for $L^{2}(w)$ if $\{\chi(x \cdot b)\}_{b \in B}$ is an orthogonal basis for $L^{2}(w)$. That is

(1) the orthogonality holds:

$$
\sum_{x \in \mathbb{Z}_{p}^{d}} \chi\left(x \cdot\left(b-b^{\prime}\right)\right) w(x)=0 \quad \forall b \neq b^{\prime}, b, b^{\prime} \in B
$$

(2) $\{\chi(x \cdot b)\}_{b \in B}$ is complete in $L^{2}(w)$ : for any $f: \mathbb{Z}_{p}^{d} \rightarrow \mathbb{C}$ there exist complex numbers $\left\{c_{b}\right\}_{b \in B}$ such that

$$
f(x)=\sum_{b \in B} c_{b} \chi(x \cdot b), \forall x \in \mathbb{Z}_{p}^{d}
$$

In this case, we say $B$ is a spectrum for $L^{2}(w)$. Notice if $w>0$ everywhere and $B$ is spectrum for $L^{2}(w)$, then we must have $B=\mathbb{Z}_{p}^{d}$. 
Lemma 4.1. Let $w: \mathbb{Z}_{p}^{d} \rightarrow[0, \infty)$. Suppose that $\sum_{x \in \mathbb{Z}_{p}^{d}} w(x)=1$. If $B$ is a spectrum for $L^{2}(w)$, then

$$
\sum_{b \in B}|\widehat{w}(x-b)|^{2}=p^{-2 d} \quad \forall x \in \mathbb{Z}_{p}^{d} .
$$

Proof. By the definition of the Fourier transform on $\mathbb{Z}_{p}^{d}$ we can write the following.

$$
\begin{aligned}
\sum_{b \in B}|\hat{w}(x-b)|^{2} & =\sum_{b \in B}\left|p^{-d} \sum_{m \in \mathbb{Z}_{p}^{d}} w(m) \chi(-m \cdot(x-b))\right|^{2} \\
& =p^{-2 d} \sum_{b \in B}\left|\sum_{m \in \mathbb{Z}_{p}^{d}} \chi(-m \cdot x) \chi(m \cdot b) w(m)\right|^{2} \\
& =p^{-2 d} \sum_{b \in B}\left|\left\langle\chi_{b}, \chi_{x}\right\rangle_{L^{2}(w)}\right|^{2} .
\end{aligned}
$$

Here, $\chi_{b}(t):=\chi(t \cdot b)=e^{2 \pi i \frac{t \cdot b}{p}}$.

By the assumption that $B$ is a spectrum for $L^{2}(w)$ and $\sum_{x \in \mathbb{Z}_{p}^{d}} w(x)=1$, the set $\left\{\chi_{b}\right\}_{b \in B}$ is an orthonormal basis for $L^{2}(w)$. Therefore, we continue as follows:

$$
\sum_{b \in B}|\hat{w}(x-b)|^{2}=p^{-2 d}\left\|\chi_{x}\right\|_{L^{2}(w)}^{2}=p^{-2 d} \sum_{m \in \mathbb{Z}_{p}^{d}} w(m)=p^{-2 d} .
$$

This completes the proof of the lemma.

Lemma 4.2 (Key lemma). Let $w: \mathbb{Z}_{p}^{d} \rightarrow[0, \infty)$ with $\operatorname{supp}(w)=E$. Assume that $B$ is a spectrum for $L^{2}(w)$. Then $w=c 1_{E}$ for some constant $c>0$.

Proof. Without loss of generality we assume that $\sum_{m \in E} w(m)=1$. Then by Lemma 4.1 we have

$$
\sum_{b \in B}|\widehat{w}(x-b)|^{2}=p^{-2 d} \quad \forall x \in \mathbb{Z}_{p}^{d} .
$$

By summing both sides of the equality over $x \in \mathbb{Z}_{p}^{d}$, we deduce the following.

$$
\begin{aligned}
p^{-d} & =\sum_{x \in \mathbb{Z}_{p}^{d}} \sum_{b \in B}|\hat{w}(x-b)|^{2} \\
& =\sum_{b \in B} \sum_{x \in \mathbb{Z}_{p}^{d}}|\hat{w}(x)|^{2} \\
& =p^{-d}|B| \sum_{m \in \mathbb{Z}_{p}^{d}} w(m)^{2} \quad \text { (by Plancherel identity (1.3)). }
\end{aligned}
$$


This implies that $\sum_{m \in \mathbb{Z}_{p}^{d}} w(m)^{2}=|B|^{-1}$. To prove $w$ is a constant function, we shall continue as follows:

$$
\begin{aligned}
1 & =\sum_{m \in E} w(m) \\
& \leq|E|^{1 / 2}\left(\sum_{m \in \mathbb{Z}_{p}^{d}} w(m)^{2}\right)^{1 / 2} \quad \text { (by Cauchy-Schwartz inequality) } \\
& =|E|^{1 / 2}|B|^{-1 / 2}=1 .
\end{aligned}
$$

The result yields and $w=c 1_{E}$ for some $c>0$.

Now we are ready to state the proof of Theorem 1.5.

Proof of Theorem 1.5. Assume that $\mathcal{G}(g, A, B)$ is a Gabor orthonormal basis for $L^{2}\left(\mathbb{Z}_{p}^{d}\right)$. Then we have

$$
\sum_{x \in \mathbb{Z}_{p}^{d}}|g(x)|^{2} \chi\left(\left(b-b^{\prime}\right) x\right)=0 \quad b \neq b^{\prime}, b, b^{\prime} \in B .
$$

With the assumption that $|E|=|B|$, the preceding equation implies that $\left\{\chi_{b}\right\}_{b \in B}$ is an orthogonal basis for $L^{2}(w)$ where $w=|g|^{2}$. By Lemma 4.2 the function $w$ must be constant on its support. This forces that $|g|=|E|^{-1 / 2} 1_{E}$, where $E$ is the support of $g$. To prove $(E, A)$ is a tiling pair, note that the translations of $E$ by elements of $A$ cover the whole space. The disjointness of the translations is a direct result of the cardinality. Indeed, $|A||E|=|A||B|=p^{d}$, and this implies that $\sum_{a \in A} 1_{E}(x-a)=1$ for all $x \in \mathbb{Z}_{p}^{d}$, thus the proof is completed.

To prove the converse, notice the assumption (ii) implies $|E|=|B|$. By (iii) and (ii), for any $(a, b) \neq\left(a^{\prime}, b^{\prime}\right)$ we get

$$
\sum_{x \in \mathbb{Z}_{p}^{d}} g(x-a) \overline{g\left(x-a^{\prime}\right)} \chi\left(\left(b-b^{\prime}\right) x\right)=0 .
$$

Thus $\mathcal{G}(g, A, B)$ is an orthonormal system in $L^{2}\left(\mathbb{Z}_{p}^{d}\right)$. The completeness is deduced from the cardinality, since by the tiling property $|A||E|=p^{d}$ and by the spectral property $|B|=|E|$.

We conclude this section by an example of a Gabor window which is not an indicator function and its support $E$ has size larger than $|B|$.

Example 4.3. Let $F:=\left\{\left(t, t^{2}\right): t \in \mathbb{Z}_{p}\right\}$. $F$ is graph of a function, thus it tiles by the subgroup $\{0\} \times \mathbb{Z}_{p}$, so it is spectral ([3]). Assume that $A$ is the tiling pair and $B$ is the spectrum for $F$ with $|A|=|B|=p$. Then $\mathcal{G}\left(|F|^{-1 / 2} 1_{F}, A, B\right)$ is an orthonormal basis for $L^{2}\left(\mathbb{Z}_{p}^{2}\right)$. Let $f=|F|^{-1 / 2} 1_{F}$ and let $g=\widehat{f}$ be the Fourier transform of $f$. Then by Lemma 2.2, $\mathcal{G}(g, B,-A)$ is an orthonormal basis for $L^{2}\left(\mathbb{Z}_{p}^{2}\right)$. A direct calculation shows that $|E|=p^{2}-p+1$ where $E=\operatorname{supp}(g)$. Indeed, $|E|>p$ and $g$ is not an indicator function. Moreover, the conclusions (ii) and (iii) of Theorem 1.5 also fail.

In Section 6 we shall present an example of a Gabor window $g$ where neither $g$ nor $\hat{g}$ is an indicator function. 


\section{Proof of Theorem 1.6}

In this section we prove Theorem 1.6.

Proof. We shall first prove (iii). Assume that $\mathcal{G}(g, A, B)$ is a Gabor orthonormal basis. Then by the orthogonality, for any $a, a^{\prime} \in A$, we have

$$
\sum_{x \in \mathbb{Z}_{p}^{d}} g(x-a) g\left(x-a^{\prime}\right)=\sum_{E+a \cap E+a^{\prime}} g(x-a) g\left(x-a^{\prime}\right)=0 .
$$

Since $g$ is positive, then we must have $g(x-a) g\left(x-a^{\prime}\right)=0$ for all $x \in E+a \cap E+a^{\prime}$. This only happens if $E+a \cap E+a^{\prime}=\emptyset$ since the functions $g(x-a)$ and $g\left(x-a^{\prime}\right)$ have support in $E+a$ and $E+a^{\prime}$, respectively. This proves the packing property for $E$. To complete the proof of (iii), assume that $W$ is a subset of $\mathbb{Z}_{p}^{d}$ such that $W \cap E+a=\emptyset$ for all $a \in A$. We show $W$ must be empty. To this end, note that $\left\langle 1_{W}, g(x-a) e_{b}\right\rangle=0$ by the assumption on $W$. Thus, by the completeness of the Gabor system, $1_{W}$ must be zero and $W$ must be empty. This completes the proof of (iii).

Next we prove (i) and (ii), simultaneously. Without loss of generality, we assume $\mathbf{0} \in A$. Then by appealing to the orthogonality of the Gabor family once again, we have

$$
\sum_{x \in \mathbb{Z}_{p}^{d}} g(x)^{2} \chi\left(x\left(b-b^{\prime}\right)\right)=0 \quad \forall b, b^{\prime} \in B, b \neq b^{\prime}
$$

By (iii), $E$ is a tiling set with respect to the $A$-translations. Thus $|E||A|=p^{d}$. On the other hand we know $|A||B|=p^{d}$. Thus, $|E|=|B|$. This, along the orthogonality of $\{\chi(b x): b \in B\}$ in $L^{2}\left(g^{2}\right)$, implies that $B$ is a spectrum for the measure $g^{2}([3])$. That is $\{\chi(b x): b \in B\}$ is an orthogonal basis for $L^{2}\left(g^{2}\right)$. By Lemma $4.2, g$ has to be constant on its support. This proves (i), thus (ii).

The converse of the theorem is due Theorem 1.5.

\section{Proof of Theorem 1.7}

In this section we provide two proofs for Theorem 1.7.

Proof. We first prove the theorem in dimension $d=2$. Indeed, we show that there is a function $g \in L^{2}\left(\mathbb{Z}_{p}^{2}\right)$ for which the assumption of the theorem as well as the properties (1) and (2) hold true.

Proof 1: Let $f \in L^{2}\left(\mathbb{Z}_{p}\right)$ such

$$
\widehat{f}(m):= \begin{cases}\frac{1}{\sqrt{p}} \sum_{t \in \mathbb{Z}_{p}} \chi\left(-m t^{2}\right) & m \neq 0 \\ 1 & \text { otherwise }\end{cases}
$$

Then $|\hat{f}(m)|=1$ for all $m \in \mathbb{Z}_{p}$, (see e.g. [6]). By the inverse Fourier formula we have

$$
\begin{aligned}
f(x) & =1+\frac{1}{\sqrt{p}} \sum_{m \neq 0} \sum_{t \in \mathbb{Z}_{p}} \chi\left(m\left(x-t^{2}\right)\right) \\
& =1-\sqrt{p}+\frac{1}{\sqrt{p}} \sum_{m \in \mathbb{Z}_{p}} \sum_{t \in \mathbb{Z}_{p}} \chi\left(m\left(x-t^{2}\right)\right) .
\end{aligned}
$$


Or,

$$
f(x)= \begin{cases}1 & \text { if } \quad x=0 \\ 1+\sqrt{p} & \text { if } \quad x \neq 0 \text { and } x R p \\ 1-\sqrt{p} & \text { if } \quad x \neq 0 \text { and } x N p\end{cases}
$$

(Here, by $x R p$ we mean $x$ is quadratic residue modulo $p$, and by $x N p$ we mean $x$ is qudratic nonresidue modulo $p$.) Let $h \in L^{2}\left(\mathbb{Z}_{p}\right)$ such that for some constant $c>0,|h(x)|=c$ for all $x \in \mathbb{Z}_{p}$. We define $g$ as a product of two functions of single variable:

$$
g\left(x_{1}, x_{2}\right):=f\left(x_{1}\right) h\left(x_{2}\right) \quad\left(x_{1}, x_{2}\right) \in \mathbb{Z}_{p} \times \mathbb{Z}_{p} .
$$

We shall choose $c>0$ such that $\|g\|_{2}=1$. For the lattices $A:=\left\{(a, 0): a \in \mathbb{Z}_{p}\right\}$ and $B:=$ $\left\{(0, b), b \in \mathbb{Z}_{p}\right\}$ it can easily be checked that $\mathcal{G}(g, A, B)$ is an orthonormal set in $L^{2}\left(\mathbb{Z}_{p}^{2}\right)$. Indeed, let $(a, 0) \in A$ and $\left(0, b_{1}\right),\left(0, b_{2}\right) \in B$. Then

$$
\sum_{x_{1}, x_{2}} g\left(x_{1}-a, x_{2}\right) \overline{g\left(x_{1}, x_{2}\right)} \chi\left(x_{2}\left(b_{1}-b_{2}\right)\right)=c^{2}\left(\sum_{x_{1}} f\left(x_{1}-a\right) \overline{f\left(x_{1}\right)}\right) \sum_{x_{2}} \chi\left(x_{2}\left(b_{1}-b_{2}\right)\right) .
$$

The second sum on the right equals to zero when $b_{1} \neq b_{2}$. When $a \neq 0$, then the sum inside the parenthesis is zero by an application of the Plancherel theorem and the fact that $|\hat{f}(m)|=1$ for all $m \in \mathbb{Z}_{p}$. This proves the orthogonality of the Gabor family $\mathcal{G}(g, A, B)$ in $L^{2}\left(\mathbb{Z}_{p}^{2}\right)$. The completeness follows by an cardinality argument.

Notice, in above $\operatorname{supp}(g)=\mathbb{Z}_{p}^{2}$ and neither $|g|$ nor $|\hat{g}|$ is constant. This completes the proof for the existence of $g$ with desired properties in dimension $d=2$. For higher dimensions we shall continue as follows:

Define $\tilde{g}\left(x_{1}, \cdots, x_{d}\right):=h_{1}\left(x_{1}\right) h_{2}\left(x_{2}\right) \cdots h_{d}\left(x_{d}\right)$, where $h_{1}$ is the function $f$ in above, and for each $2 \leq i \leq d$ we have $\left|h_{i}(m)\right|=c_{i}, c_{i}>0$. Take $\tilde{A}:=\left\{(a, 0, \cdots, 0): a \in \mathbb{Z}_{p}\right\}$ and $\tilde{B}:=$ $\left\{\left(0, b_{1}, \cdots, b_{d-1}\right), b_{i} \in \mathbb{Z}_{p}\right\}$. The orthogonality of $\mathcal{G}(\tilde{g}, \tilde{A}, \tilde{B})$ can be obtained in the same way as in (6.3) and the following argument. The completeness holds by the cardinality.

Proof 2. Here, we replace the Gaussian summation (6.1) above and function $f$ by the following function. Take $f \in L^{2}\left(\mathbb{Z}_{p}^{2}\right)$ such that $\hat{f}(m)=1$ for all $m \neq 0$ and $\hat{f}(0)=-1$. Then it is clear that $|\hat{f}(m)|=1$, thus the orthogonality of the Gabor set $\mathcal{G}(g, A, B)$ holds for same $A$ and $B$ as above. Then, by the inverse of Fourier transform, we have $f(x)=p-2$ if $x \neq 0$ and $f(0)=-2$. Note that neither $\hat{f}$ nor $f$ is constant multiple of a characteristic function, therefore the function $g$, constructed as in (6.2), is a Gabor window function which satisfies the properties (1) and (2). For the higher dimensions, we repeat the argument as in Proof 1.

\section{Proof of Theorem 1.8}

This section provides a proof for Theorem 1.8.

Proof. For the sake of simplicity, we prove the theorem only for the case $d=2$. The proof for higher dimensions is similar.

" $\Longrightarrow$ " For the proof of (a), let $a \neq 0$. Then by mutual orthogonality of $\mathcal{G}(g, A, B)$ in $L^{2}\left(\mathbb{Z}_{p}^{2}\right)$, for any $b \in \mathbb{Z}_{p}$ we get

$$
\sum_{x_{2} \in \mathbb{Z}_{p}}\left(\sum_{x_{1} \in \mathbb{Z}_{p}} g\left(x_{1}-a, x_{2}\right) \overline{g\left(x_{1}, x_{2}\right)}\right) \chi\left(x_{2} b\right)=0
$$


The uniqueness of the Fourier transform implies that for all $a \neq 0$ we have

$$
\sum_{x_{1} \in \mathbb{Z}_{p}} g\left(x_{1}-a, x_{2}\right) \overline{g\left(x_{1}, x_{2}\right)}=0 \quad \forall x_{2} \in \mathbb{Z}_{p} .
$$

If we let $g_{y}: \mathbb{Z}_{p} \rightarrow \mathbb{C}$ define $g_{y}(x):=g(x, y)$, then we can rewrite the preceding equation as

$$
\sum_{x_{1} \in \mathbb{Z}_{p}} g_{x_{2}}\left(x_{1}-a\right) \overline{g_{x_{2}}\left(x_{1}\right)}=0 \quad \forall x_{2} \in \mathbb{Z}_{p} .
$$

By applying the Parseval identity to the equation $(*)$ in variable $x_{1}$, for any $x_{2} \in \mathbb{Z}_{p}$ we obtain

$$
\sum_{m \in \mathbb{Z}_{p}}\left|\hat{g}_{x_{2}}(m)\right|^{2} \chi(-a m)=0 \quad \forall a \neq 0 .
$$

The relation (7.1) forces $\left|\hat{g}_{x_{2}}\right|$ to be a constant function. Or equivalently,

$$
\left|\sum_{x_{1}} g\left(x_{1}, x_{2}\right) \chi\left(-x_{1} \cdot m\right)\right|=\text { constant } \quad \forall m \in \mathbb{Z}_{p},
$$

hence the proof of (a) is completed. (Notice this constant depends only on $x_{2}$.)

To prove (b), let $b \neq 0$. The orthogonality assumption implies that

$$
\sum_{\left(x_{1}, x_{2}\right) \in \mathbb{Z}_{p}^{2}}\left|g\left(x_{1}, x_{2}\right)\right|^{2} \chi\left(-b x_{2}\right)=0 .
$$

Thus for $G\left(x_{2}\right):=\sum_{x_{1}}|g|^{2}\left(x_{1}, x_{2}\right)$, the existing equation means that $\hat{G}(b)=0$ for all $b \neq 0$. This implies that $G$ is constant, indeed, $G(x)=\hat{G}(0), x \in \mathbb{Z}_{p}$, and hence (b) follows.

"£": To prove the converse, assume that the conditions (a) and (b) hold. By (b) we have

$$
\sum_{x_{1}} g\left(x_{1}, x_{2}\right) \overline{g\left(x_{1}, x_{2}\right)}=\text { "constant in } x_{2} "
$$

Then for any $b \neq 0$

$$
\sum_{x_{2}} \sum_{x_{1}} g\left(x_{1}, x_{2}\right) \overline{g\left(x_{1}, x_{2}\right)} \chi\left(b x_{2}\right)=(\text { constant }) \sum_{x_{2}} \chi\left(b x_{2}\right)=0 .
$$

This is equivalent to saying that

$$
\left\langle g, \chi_{(0, b)} g\right\rangle_{L^{2}\left(\mathbb{Z}_{p}^{2}\right)}=0 \quad \forall b \neq 0 .
$$

Here, $\chi_{(m, n)} f(x, y):=f(x, y) \chi(m x+n y)$.

On the other hand, (a) holds. Then for any $x_{2} \in \mathbb{Z}_{p}$

$$
\left|\hat{g}_{x_{2}}(m)\right|=c \quad \forall m \in \mathbb{Z}_{p}
$$

where $c:=c\left(x_{2}\right)$ is a constant depending on $x_{2}$. Thus for $a \neq 0$

$$
\sum_{m}\left|\hat{g}_{x_{2}}(m)\right|^{2} \chi(-a m)=0 .
$$


This is equivalent to say that $\left\langle\hat{g}_{x_{2}}, \widehat{\tau_{a} g_{x_{2}}}\right\rangle_{L^{2}\left(\mathbb{Z}_{p}\right)}=0$, where $\tau_{a} f(x):=f(x-a)$. Thus, by the Parseval identity

$$
\left\langle g_{x_{2}}, \tau_{a} g_{x_{2}}\right\rangle_{L^{2}\left(\mathbb{Z}_{p}\right)}=0 \quad \text { for } a \neq 0
$$

This immediately leads to

$$
\left\langle g, \chi_{(0, b)} \tau_{(a, 0)} g\right\rangle_{L^{2}\left(\mathbb{Z}_{p}^{2}\right)}=0 \quad \forall a \neq 0 .
$$

From the orthogonality relations in (7.2) and (7.3) we deduce that the set $\mathcal{G}(g, A, B)$ is mutual orthogonal in $L^{2}\left(\mathbb{Z}_{p}^{2}\right)$. The completeness is a conclusion of the cardinality.

Example 7.1. Here we construct an example of Gabor window satisfying (a) and (b) in Theorem 1.8 and $g$ is not an indicator function. Define $g: \mathbb{Z}_{p}^{2} \rightarrow \mathbb{C}$ as follows: For any $(k, r) \in \mathbb{Z}_{p}^{2}$

$$
g(k, r):= \begin{cases}1 & \text { if } k=0 \\ 1+\sqrt{p} & \text { if } k \neq 0 \text { and } k R p \\ 1-\sqrt{p} & \text { if } k \neq 0 \text { and } k N p .\end{cases}
$$

(In above, by $k R p$ and $k N p$ we mean $k$ is quadratic residue or non-residue ( $\bmod p$ ), respectively.) The definition indicates that the all rows of the matrix $[g(k, r)]_{p \times p}$ are the same, where $(k, r) \in$ $\mathbb{Z}_{p} \times \mathbb{Z}_{p}$ and $g(k, r)$ indicates the entry in $k$-th row and $r$-th column. Therefore, $g$ satisfies both conditions (a) and (b) in Theorem 1.8. Moreover, for any $(m, n) \in \mathbb{Z}_{p}^{2}$

$$
\widehat{g}(m, n):= \begin{cases}1 & \text { if } m=0, n=0 \\ p^{-1 / 2} \sum_{t \in \mathbb{Z}_{p}} \chi\left(-m t^{2}\right) & \text { if } m \neq 0, n=0 \\ 0 & \text { elsewhere. }\end{cases}
$$

Indeed, it is easy to see that $\widehat{g}(0,0)=1$. For $m \neq 0$, by the definition of Fourier transform, a direct calculation shows that

$$
\widehat{g}(m, 0)=p^{-1 / 2} \sum_{k \in \mathbb{Z}_{p}}\left|Z\left(t^{2}-k\right)\right| \chi(-m k),
$$

where $\left|Z\left(t^{2}-k\right)\right|=1$ if $k=0,\left|Z\left(t^{2}-k\right)\right|=2$ if $k R p$ and $k \neq 0$, and $\left|Z\left(t^{2}-k\right)\right|=0$ if $k N p$ and $k \neq 0$.

Notice that neither $g$ nor $\widehat{g}$ is an indicator function.

We conclude the paper with the following open problems:

Question. Is there any Gabor window $g \in L^{2}\left(\mathbb{Z}_{p}^{d}\right)$ with support $E$ for which the all following properties hold simultaneously?

- $g$ is not in form of product of functions of single variable.

- $g$ is not positive.

- $|g| \neq c 1_{E}$, i.e. is not multiple of any characteristic function.

- $|\hat{g}| \neq c 1_{F}$, i.e. is not multiple of any characteristic function.

- $|E| \neq|B|$ (thus $E$ does not tile).

- $E$ does not tile (thus $|E| \neq|B|$ ). 
Problem. Classify all Gabor orthonormal bases of the form

and $S \subset \mathbb{Z}_{p}^{2 d}, d \geq 1$.

$$
\mathcal{G}(g, S):=\{g(x-a) \chi(x \cdot b):(a, b) \in S\}
$$

As mentioned earlier, Lemma 2.1 provides a characterization of Gabor orthonormal bases for dimension $d=1$ when $S$ is separable, i.e. $S=A \times B$.

\section{REFERENCES}

[1] C. Aten, B. Ayachi, E. Bau, D. FitzPatrick, A. Iosevich, H. Liu, A. Lott, I. MacKinnon, S. Maimon, S. Nan, J. Pakianathan, G. Petridis, C. Rojas Mena, A. Sheikh, T. Tribone, J. Weill, C. Yu, Tiling sets and spectral sets over finite fields, Journal of Functional Analysis Volume 273, Issue 8, 15 October 2017, Pages 2547-2577 2

[2] B. Fuglede, Commuting self-adjoint partial differential operators and a group theoretic problem, J. Funct. Anal. 16 (1974), 101-121. 2

[3] A. Iosevich, A. Mayeli and J. Pakianathan, The Fuglede Conjecture holds in $\mathbb{Z}_{p} \times \mathbb{Z}_{p}$, Analysis \& PDE, Vol. 10, No. 4, 2017. 1, 2, 6, 7

[4] M. Kolountzakis and M. Matolcsi, Tiles with no spectra, Forum Math. 18 (2006), no. 3, 519-528. 2

[5] Y. Liu, Y. Wang, The Uniformity of Non-Uniform Gabor Bases, Advances in Computational Mathematics February 2003, Volume 18, Issue 2, pp 345-355. 2

[6] E. Stein, R. Shakarchi, Fourier Analysis: An Introduction, Princeton Lectures in Analysis, Princeton University Press, 2003. 7

[7] T. Tao, Fuglede's conjecture is false in 5 and higher dimensions, Math. Res. Lett. 11 (2004), no. 2-3, 251-258. 2

Alex Iosevich, Department of Mathematics, University of Rochester, Rochester, Ny, USA

E-mail address: iosevich@math.rochester.edu

Mihalis Kolountzakis,, Department of Mathematics and Applied Mathematics, University of Crete, Crete Greece

E-mail address: kolount@gmail.com

Yu. Lyubarskit, Department of Mathematical Sciences, Norwegian University of Science and TechNOLOGY, TROHDHEIM, NORWAY

E-mail address: yurii.lyubarskii@math.ntnu.no

Azita Mayeli, Department of Mathematics and Computer Science, Queensborough and The Graduate Center, City University of New York, USA

E-mail address: AMayeli@gc.cuny.edu

Jonathan Pakianathan, Department of Mathematics, University of Rochester, Rochester, Ny, USA

E-mail address: jonpak@math.rochester.edu 